\title{
A comparison of oxycodone and fentanyl in intravenous patient-controlled analgesia after laparoscopic hysterectomy
}

\author{
Nan-Seol Kim ${ }^{1}$, Kyu Sik Kang ${ }^{1}$, Sie Hyeon Yoo ${ }^{1}$, Jin Hun Chung ${ }^{1}$, \\ Ji-Won Chung ${ }^{1}$, Yonghan Seo ${ }^{1}$, Ho-Soon Chung ${ }^{1}$, Hye-Rim Jeon ${ }^{1}$, \\ Hyung Youn Gong ${ }^{1}$, Hyun-Young Lee ${ }^{1}$, and Seong-Taek Mun ${ }^{2}$ \\ Departments of ${ }^{1}$ Anesthesiology and Pain Medicine, ${ }^{2}$ Obstetrics and Gynecology, Soonchunhyang University \\ Cheonan Hospital, Soonchunhyang Univertisy College of Medicine, Cheonan, Korea
}

Background: We planned to compare the effect of intravenous oxycodone and fentanyl on post-operative pain after laparoscopic hysterectomy.

Methods: We examined 60 patients were randomized to postoperative pain treatment with either oxycodone $(\mathrm{n}=30$, Group O) or fentanyl ( $\mathrm{n}=30$, Group F). The patients received $10 \mathrm{mg}$ oxycodone $/ 100 \mu \mathrm{g}$ fentanyl with ketorolac $30 \mathrm{mg}$ before the end of anesthesia and then continued with patient-controlled analgesia for $48 \mathrm{~h}$ postoperatively.

Results: The accumulated oxycodone consumption was less than fentanyl during 8, 24 and $48 \mathrm{~h}$ postoperatively. Numeric rating score of Group O showed significantly lower than that of Group F during $30 \mathrm{~min}, 2,4,8$ and 24 h postoperatively. The incidences of adverse reactions were similar in the two groups, though the incidence of nausea was higher in the Group O during the 24 and $48 \mathrm{~h}$ postoperative period.

Conclusions: Oxycodone IV-PCA was more advantageous than fentanyl IV-PCA for laparoscopic hysterectomy in view of accumulated oxycodone consumption, pain control and cost beneficial effect. However, patient satisfaction was not good in the group $\mathrm{O}$ compared to group $\mathrm{F}$.

Key Words: Fentanyl, Oxycodone, Postoperative pain.

Corresponding author: Kyu Sik Kang, M.D., Ph.D.

Department of Anesthesiology and Pain Medicine, Soonchunhyang University Cheonan Hospital, Soonchunhyang Univertisy College of Medicine, 31, Suncheonhyang 6-gil, Dongnam-gu, Cheonan 330-721, Korea

Tel: 82-41-570-2722, Fax: 82-41-573-3559

E-mail: ksjsk@schca.ac.kr

Received: November 26, 2014.

Revised: December 24, 2014.

Accepted: December 31, 2014.

Korean J Anesthesiol 2015 June 68(3): 261-266 http://dx.doi.org/10.4097/kjae.2015.68.3.261

\section{Introduction}

While several methods can be used for postoperative pain management, intravenous patient-controlled analgesia (IV-PCA) is the most common, as it provides efficacious postoperative analgesia and high patient satisfaction with minimal sedation and complications [1].

The most commonly-used drugs for IV-PCA are opioids, including morphine. Morphine has been the single most studied and used drug for IV-PCA and is therefore considered the gold standard analgesic for IV-PCA. However, due to its hydrophilic

(c) This is an open-access article distributed under the terms of the Creative Commons Attribution Non-Commercial License (http://creativecommons.org/ licenses/by-nc/4.0/), which permits unrestricted non-commercial use, distribution, and reproduction in any medium, provided the original work is properly cited. 
property, morphine cannot cross the blood-brain barrier easily, delaying the onset of effects. Moreover, its active metabolite, morphine-6-glucuronide, lowers the metabolism of patients with impaired renal functions, which may result in unexpected complications such as respiratory depression $[1,2]$.

Our hospital uses fentanyl, which is $80-100$ times more potent than morphine. In addition, its high lipid solubility reduces the onset time, making it suitable for IV-PCA [1,2].

Dihydrohydroxycodeinone (oxycodone) is a thebaine derivative and is structurally similar to morphine. It is a potent $\mu$-agonist, with a potency comparable to that of morphine [3]. In Finland, parenteral oxycodone has been used in acute pain control since the 1960s [4]; nevertheless, there have been few studies on the analgesic efficacy of fentanyl and oxycodone for IV-PCA in postoperative pain management [5].

This study therefore set out to compare the analgesic efficacy, side effects, and patient satisfaction ratings of fentanyl and oxycodone, two common analgesics used in our hospital for IVPCA following laparoscopic hysterectomy.

\section{Materials and Methods}

This study was conducted after receiving approval from our hospital's Institutional Review Board. It involved 60 adult patients between the ages of 18-70 who had been classified as American Society of Anesthesiologists (ASA) physical status 1 and 2, were scheduled for laparoscopic hysterectomy at our hospital, and had requested IV-PCA after the operation.

Patients with a history of bleeding tendencies, hepatitis and renal failure, those with habitual sedative or other drug use, those with mental diseases, and those not suitable for IV-PCA, were excluded from the study.

The purpose of the study, PCA instructions, numeric rating scale (NRS) calculation method, and side effects were explained

Table 1. Demographic Data and Anesthesia Characteristics

\begin{tabular}{lcc}
\hline & $\begin{array}{c}\text { Group F } \\
(\mathrm{n}=30)\end{array}$ & $\begin{array}{c}\text { Group O } \\
(\mathrm{n}=30)\end{array}$ \\
\hline Age $(\mathrm{yr})$ & $48.1 \pm 5.3$ & $45.6 \pm 5.6$ \\
ASA physical status I/II & $22 / 8$ & $24 / 6$ \\
Smoker/nonsmoker & $4 / 26$ & $4 / 26$ \\
History of motion sickness or PONV & $12(40 \%)$ & $13(43 \%)$ \\
Height $(\mathrm{cm})$ & $158.0 \pm 4.4$ & $156.6 \pm 4.7$ \\
Weight $(\mathrm{kg})$ & $58.8 \pm 8.5$ & $58.8 \pm 9.8$ \\
Duration of surgery (min) & $75.2 \pm 24.9$ & $73.3 \pm 23.0$ \\
Total propofol consumption $(\mathrm{mg})$ & $760.9 \pm 222.3$ & $697.0 \pm 157.4$ \\
Total remifentanil consumption $(\mu \mathrm{g})$ & $679.2 \pm 191.0$ & $605.6 \pm 208.9$ \\
\hline
\end{tabular}

Values are expressed as mean \pm SD or number of patients (\%). No significant differences between the groups. Group F: fentanyl group, Group O: oxycodone group, PONV: postoperative nausea and vomiting. to the patients one day before the surgery, and written consent forms were collected. The patients were randomly divided into two groups: a group using fentanyl for the IV-PCA (Group F, $\mathrm{n}=30$ ) and a group using oxycodone for the IV-PCA (Group O, $n=30$ ). There was no statistically significant difference between the two groups in terms of patients' age, height, weight, duration of operation, ASA physical status, smoking habits, and intensity of motion sickness (Table 1).

The patients were not given any preanesthetic medication, and venous access was achieved with an $18 \mathrm{G}$ needle before they were moved to the operating room. Upon arrival in the operating room, the patients were connected to an electrocardiogram monitor, noninvasive blood pressure monitoring device, and pulse oximeter. After connecting the monitoring devices, 0.2 mg of glycopyrrolate (Mobinul ${ }^{\mathrm{TM}}$, Myungmoon, Seoul, Korea) was administered intravenously and preoxygenation was performed with $100 \%$ oxygen at $8 \mathrm{~L} / \mathrm{min}$. Then, an Orchestra ${ }^{\circledR}$ Base Primea (Fresenius Vial, Brezins, France) pump was used to initiate the administration of remifentanil (Ultiva ${ }^{\mathrm{TM}}$, GlaxoSmithKline, Verona, Italy) with an effect-site concentration of $4.0 \mathrm{ng} / \mathrm{ml}$. After the targeted concentration of remifentanil was achieved, propofol (Presofol MCT 2\% ${ }^{\mathrm{TM}}$, Fresenius Kabi, Graz, Austria) was administered with an effect-site concentration of $4 \mu / \mathrm{ml}$. Upon confirming the loss of response to verbal orders, $0.8 \mathrm{mg} / \mathrm{kg}$ of rocuronium bromide (Esmeron ${ }^{\mathrm{TM}}$, Organon, The Netherlands) was intravenously administered, and endotracheal intubation was performed 90 seconds later. Mechanical ventilation was then initiated to oxygenate the patient with $\mathrm{FIO}_{2}$ of 0.4 and maintain an end-tidal carbon dioxide of $30-40 \mathrm{mmHg}$. The effect-site concentrations of propofol and remifentanil were controlled in order to maintain a change of less than $20 \%$ in the blood pressure and heart rate from the preanesthetic levels and to maintain a proper depth of anesthesia.

At the skin suturing stage, IV-PCA device (Baxter, Healthcare Corporation, Deerfield, IL, USA) was connected to the patient. Group F was administered $100 \mu \mathrm{g}$ of fentanyl and 30 $\mathrm{mg}$ of ketorolac, and Group $\mathrm{O}$ was administered $10 \mathrm{mg}$ of oxycodone and $30 \mathrm{mg}$ of ketorolac for postoperative pain relief. Moreover, $0.3 \mathrm{mg}$ of ramosetron was administered in order to prevent postoperative nausea and vomiting, the most common side effects. Upon completion of the surgery, the administration of anesthetics was stopped and the neuromuscular blockade was reversed with pyridostigmine and glycopyrrolate. Extubation was performed after confirming the recovery of spontaneous breathing.

The concentrations for the IV-PCA were determined after conducting a few concentration trials. Group F was administered a $100 \mathrm{ml}$-mixture of $700 \mu \mathrm{g}$ of fentanyl, $150 \mathrm{mg}$ of ketorolac, and $0.6 \mathrm{mg}$ of ramosetron with a normal saline solution, with a release rate of $14 \mu \mathrm{g} / \mathrm{hr}$, a volume per administration of 0.5 
$\mathrm{ml}$, and a lockout interval of 15 minutes. Group $\mathrm{O}$ was administered a $100 \mathrm{ml}$-mixture of $70 \mathrm{mg}$ of oxycodone (conversion dose ratio of fentanyl to oxycodone, $1: 100$ ), $150 \mathrm{mg}$ of ketorolac, and $0.6 \mathrm{mg}$ of ramosetron with a normal saline solution, with a release rate of $1.4 \mathrm{mg} / \mathrm{hr}$, a volume per administration of $0.5 \mathrm{ml}$, and a lockout interval of 15 minutes over 48 hours. If patient's side effects became too severe, we interrupted the IV-PCA and reconnected later - once the symptoms had subsided or the patient requested it. A double-bind test was performed in order to test the drugs for the IV-PCA.

The amount of analgesics consumed, administration of additional analgesics, level of pain, level of sedation, and side effects were evaluated and recorded 30 minutes, 2 hours, 4 hours, 8 hours, and 24 hours and 48 hours after the surgery.

The level of pain was measured using the NRS, with 0 indicating no pain and 100 unbearable pain. When a patient requested further analgesics even with the administration of a preset dose, $50 \mu \mathrm{g}$ of fentanyl were additionally administered.

For the level of sedation, a score of 2 was given if the patient's level of consciousness was as clear as before the surgery, a score of 1 if the patient was drowsy and verbal stimulation was enough to awake him/her, and a score of 0 if the patient was drowsy and had to be physically stimulated to wake up.

The side effects observed were postoperative nausea and vomiting (PONV), dizziness, pruritus (itching), headache, and respiratory depression.

The patients' satisfaction was assessed 48 hours after the surgery, and the rating levels were divided into very satisfied, satisfied, neutral, and dissatisfied.

Previous studies were examined in order to determine the effective number of subjects [6]. The mean difference of the pri-

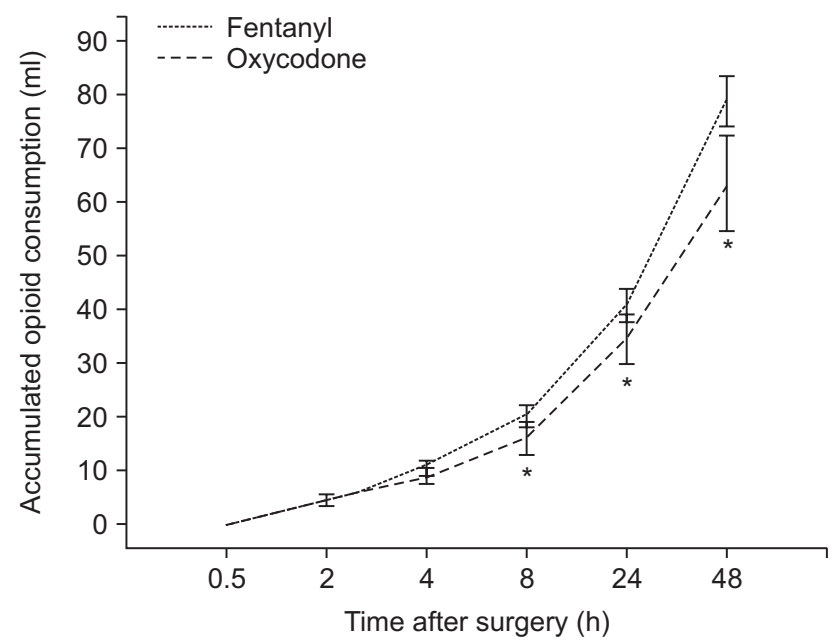

Fig. 1. Accumulated Fentanyl and Oxycodone consumption (ml) $0.5-48$ h postoperatively (mean and $95 \%$ confidence interval). $* \mathrm{P}<0.05$ between two groups. mary endpoints between the groups for a clinically significant effect size was assumed to be $61 \mathrm{mg}$, and the standard deviations for groups $\mathrm{F}$ and $\mathrm{O}$ were assumed to be $50 \mu \mathrm{g}$ and $86 \mathrm{mg}$, respectively. Subsequently, it was determined that the required number of subjects per group was 21 when the a value (level of significance) was 0.05 (two-sided) and the power 1 - $\beta$ was 0.8 .

SPSS for Windows (version 14, SPSS Inc., Chicago, IL, USA) was used for the statistical analysis. The calculated data were recorded in the form of mean \pm standard deviation. The demographic data, volume of IV-PCA consumption over 48 hours, and comparison of the level of pain at rest and while coughing, were assessed and analyzed with a generalized estimating equation. A comparative analysis of the frequency of side effects and satisfaction between the groups was performed using Pearson's chi-square test or Fisher's exact test. A P value under 0.05 was considered statistically significant.

\section{Results}

There was no statistically significant difference between the two groups in the volume of IV-PCA consumed 30 minutes, 2 hours and 4 hours after the surgery, but the volume of IV-PCA consumed 8 hours, 24 hours, and 48 hours after the surgery was significantly lower in Group O than in Group F (Fig. 1).

There was no significant difference between the two groups in terms of pain score at rest and while coughing 48 hours after the surgery. However, 30 minutes, 2 hours, 4 hours, 8 hours, and 24 hours post-surgery, the pain scores at rest and while coughing were significantly lower in Group O than in Group F (Figs. 2 and 3).

In Group F, the level of NRS pain at rest 2 hours after the

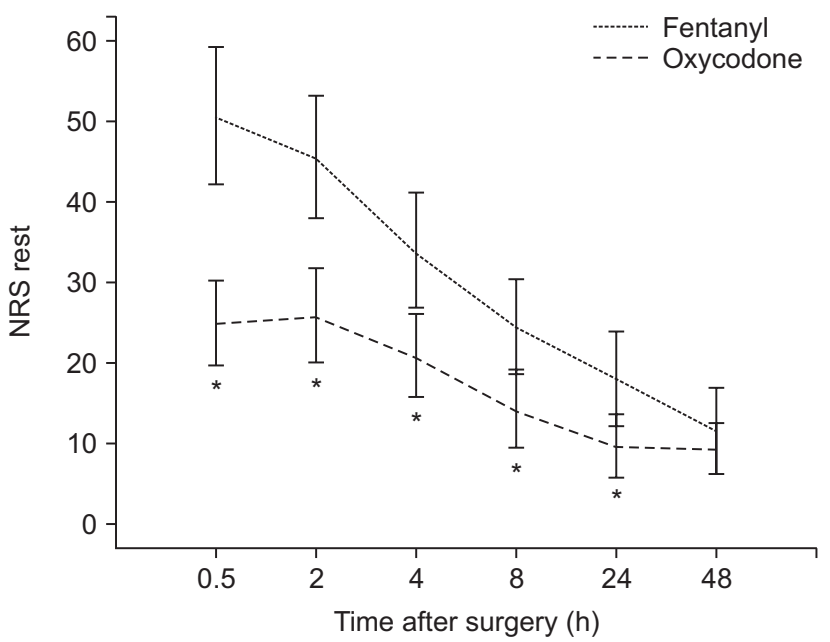

Fig. 2. Pain score by numeric rating scale (NRS, $0-100 \mathrm{~mm}$ ) at rest $0.5-48 \mathrm{~h}$ postoperatively (mean and $95 \%$ confidence interval). $* \mathrm{P}<0.05$ between two groups. 


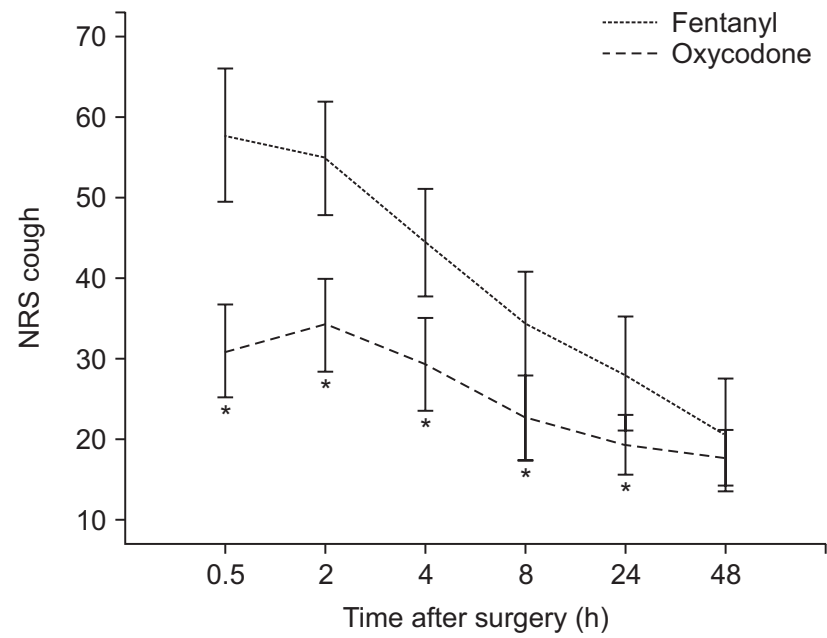

Fig. 3. Pain score by numeric rating scale (NRS, $0-100 \mathrm{~mm}$ ) when coughing $0.5-48 \mathrm{~h}$ postoperatively (mean and $95 \%$ confidence interval). $* \mathrm{P}<0.05$ between two groups.

Table 2. Incidence Rate (\%) of Postoperative Nausea and Vomiting

\begin{tabular}{clllll}
\hline \multirow{2}{*}{$\begin{array}{c}\text { Time after } \\
\text { surgery }\end{array}$} & \multicolumn{2}{c}{$\begin{array}{c}\text { Group F } \\
(\mathrm{n}=30)\end{array}$} & & \multicolumn{2}{c}{$\begin{array}{c}\text { Group O } \\
(\mathrm{n}=30)\end{array}$} \\
\cline { 2 - 3 } \cline { 5 - 6 } & Nausea & Vomiting & & Nausea & Vomiting \\
\hline $0.5 \mathrm{~h}$ & $1(3.3 \%)$ & $0(0 \%)$ & & $1(3.3 \%)$ & $0(0 \%)$ \\
$2 \mathrm{~h}$ & $3(10 \%)$ & $1(3.3 \%)$ & & $4(13.3 \%)$ & $0(0 \%)$ \\
$4 \mathrm{~h}$ & $6(20 \%)$ & $1(3.3 \%)$ & & $14(46.7 \%)$ & $1(3.3 \%)$ \\
$8 \mathrm{~h}$ & $6(20 \%)$ & $0(0 \%)$ & & $13(43.3 \%)$ & $2(6.7 \%)$ \\
$24 \mathrm{~h}$ & $4(13.3 \%)$ & $2(6.7 \%)$ & & $12(40.0 \%)^{*}$ & $4(13.3 \%)$ \\
$48 \mathrm{~h}$ & $4(13.3 \%)$ & $1(3.3 \%)$ & $12(40.0 \%)^{*}$ & $3(10.0 \%)$ \\
\hline
\end{tabular}

Values are expressed as number of patients (\%). Group F: Fentanyl group, Group $\mathrm{O}$ : oxycodone group. ${ }^{*} \mathrm{P}<0.05$ between two groups.

surgery was not significantly different from that 30 minutes after the surgery. However, the level of pain was significantly lower at all other time points $(\mathrm{P}<0.05)$. In Group $\mathrm{O}$, the levels of NRS pain at rest 2 hours and 4 hours after the surgery were not significantly different from that 30 minutes after the surgery. However, the level of pain was statistically significantly lower 8 hours, 24 hours, and 48 hours after the surgery. Moreover, in Group F, the level of NRS pain while coughing 2 hours after the surgery was not significantly different from that 30 minutes after the surgery. However, the level of pain while coughing was significantly lower at all other time points $(\mathrm{P}<0.05)$. In Group $\mathrm{O}$, the levels of NRS pain while coughing 2 hours, 4 hours, and 8 hours after the surgery were not significantly different from that 30 minutes after the surgery. However, the level of pain while coughing was statistically significantly lower 24 hours and 48 hours post-surgery $(\mathrm{P}<0.05)$.

The additional administration of analgesics 30 minutes after the surgery was significantly higher in Group F than in Group O,
Table 3. Incidence Rate (\%) of Adverse Events

\begin{tabular}{lll}
\hline & $\begin{array}{l}\text { Group F } \\
(\mathrm{n}=30)\end{array}$ & $\begin{array}{l}\text { Group O } \\
(\mathrm{n}=30)\end{array}$ \\
\hline Dizziness & $6(20 \%)$ & $8(26.7 \%)$ \\
Headache & $4(13.3 \%)$ & $4(13.3 \%)$ \\
Pruritis & $3(10 \%)$ & $4(13.3 \%)$ \\
Respiratory depression & $0(0 \%)$ & $0(0 \%)$ \\
\hline
\end{tabular}

Values are expressed as number of patients (\%). No significant differences between the two groups. Group F: Fentanyl group, Group O: oxycodone group.

Table 4. Patient Satisfaction at Postoperative 48 Hours

\begin{tabular}{lcr}
\hline & $\begin{array}{c}\text { Group F } \\
(\mathrm{n}=30)\end{array}$ & \multicolumn{1}{c}{$\begin{array}{c}\text { Group O } \\
(\mathrm{n}=30)\end{array}$} \\
\hline Very satisfied & $9(30.0 \%)$ & $5(16.7 \%)$ \\
Satisfied & $16(53.3 \%)$ & $15(50.0 \%)$ \\
Neutral & $5(16.7 \%)$ & $6(20.0 \%)$ \\
Dissatisfied & $0(0 \%)$ & $4(13.3 \%)$ \\
\hline
\end{tabular}

Values are expressed as number of patients (\%). No significant differences between the two groups. Group F: Fentanyl group, Group O: oxycodone group.

as 14 subjects in Group F against only 1 in Group $\mathrm{O}$ requested additional analgesics $(\mathrm{P}<0.05)$.

There was no statistically significant difference between the groups in terms of nausea 30 minutes, 2 hours, 4 hours, and 8 hours after the surgery, whereas Group O showed a significantly higher incidence of nausea than Group F 24 hours and 48 hours after the surgery (Table 2). On the other hand, there was no statistically significant difference between the two groups in terms of vomiting.

There were no statistically significant intergroup differences for all other side effects, and no one showed signs of respiratory depression (Table 3). Three subjects in Group F (10.3\%) and 5 subjects in Group O (16.7\%) had a sedation score of 130 minutes post-surgery while all other patients had a score of 2; therefore, there was no significant intergroup difference in terms of sedation.

The patients' satisfaction was assessed 48 hours after the surgery, and there was no statistically significant difference between the two groups (Table 4).

\section{Discussion}

The purpose of this study was to compare the total consumption, analgesic efficacy, side effects, and patient satisfaction ratings for two IV-PCA drugs, the commonly-used fentanyl and newly-introduced IV oxycodone, over a period of 48 hours post-surgery.

An adequate analgesic effect can only be achieved through IV-PCA when the most appropriate analgesic is selected and its 
dose and lockout intervals are properly controlled. Therefore, clinical physicians must take extra precautions when determining the dosage of the chosen analgesic, as an insufficient amount will not generate effective analgesic effects, and an excessive amount will cause side effects.

Opioids, the most commonly used type of analgesics for IVPCA, are full $\mu$-receptor binding agonists and have no analgesic ceiling. Nevertheless, they have a clinical ceiling in terms of side effects such as respiratory depression, which may prevent clinicians from administering the entire required dose [1]. Moreover, while opioids are effective for alleviating pain at rest, their effect in treating movement-evoked pain is minimal. This is due to the fact that opioids have little effect on changing neuroplasticity or reversing central sensitization. On the other hand, some reports have suggested that the administration of local anesthetics, COX- 2 inhibitors, $\alpha 2$ agonists, and N-methyl-D-aspartic acid receptor antagonists can be effective in reducing movementevoked pain by preventing central sensitization [7]. That being said, in this study, ketorolac was included in the IV-PCA mixture for the purpose of mitigating movement-evoked pain.

Fentanyl is a 4-amilidopiperidien compound with a high lipid solubility, which greatly reduces the onset time of the analgesic effect. In fact, the onset time of fentanyl is 30 seconds, with its maximum effect reached 5 minutes after administration. Fentan$\mathrm{yl}$ is also a potent and ideal drug for IV-PCA, as its redistribution is rapid and wide with a short duration, and it does not produce the active metabolites that cause respiratory depression $[1,2]$.

The recently-introduced intravenous (IV) oxycodone is a thebaine-derived, semi-synthetic opioid that is widely used in the treatment of both acute and chronic pain, and has been commonly administered orally due to its high oral bioavailability $(60-87 \%)[3,8]$. The Ministry of Food and Drug Safety approved IV oxycodone for IV-PCA use in 2013. However, the safe dose conversion ratio of oxycodone to fentanyl (as a more commonly-used opioid) is yet to be established. Oxycodone is metabolized in the liver through its principal metabolic pathway, the cytochrome P 450 (CYTP) 3A enzyme, and second metabolic pathway (representing about 10\%), CYP2D6. The central opioid effects of oxycodone are regulated by the parent drug, and the contributions from its circulating oxidative and reductive metabolites are considered negligible $[9,10]$. Oxycodone has similar side effects as other opioids, namely, nausea, constipation and drowsiness, as well as vomiting, itching, and dizziness. Compared to morphine, oxycodone rarely induces nausea, hallucinations or pruritus [8]. It has a similar analgesic onset time as fentanyl (about $2-3$ minutes), with a slightly longer duration (t1/2: 4 h 52 min vs. 3 h 39 min) [5].

Considering the lack of guidelines for the direct conversion dose ratio of IV oxycodone to IV fentanyl, this study reviewed previous research in order to identify the used ratios. Prior stud- ies were found to have used a morphine to oxycodone ratio of $1: 1[11,12]$ and fentanyl to morphine ratio of $1: 100[2,13]$. On that basis, we calculated a workable fentnayl to oxycodone ratio of $1: 100$.

Koch et al. [5] previously compared the consumption amounts and side effects of IV fentanyl and oxycodone as used for postoperative analgesia in patients undergoing laparoscopic cholesystectomy for 2 hours following arrival in the recovery room. They reported that the oxycodone group experienced better analgesic effects than the fentanyl group (i.e., more effective than the $100: 1$ ratio), and that the total amount of oxycodone consumed was $15 \mathrm{mg}$ vs $200 \mu \mathrm{g}$ of fentanyl (a ratio of about $75: 1$ ). They also reported that although the difference was statistically insignificant, the oxycodone group experienced more side effects. The present study echoes these results. There was a significant difference between the oxycodone group and fentanyl group in terms of the total amount of analgesics consumed from 8 hours after the surgery, and the oyxcodone group exhibited statistically significantly lower pain scores at rest and while coughing than the fentanyl group up to 24 hours after the surgery (Figs. 2 and 3).

Oxycodone presents a notable superiority over fentanyl in terms of cost; the IV-PCA using oxycodone was much cheaper (by about 40\%) than the IV-PCA using fentanyl (fentanyl: 16,167 Korean Won, vs oxycodone: 9,824 Korean Won).

The most common postoperative side effects of IV-PCA are nausea and vomiting. The high-risk factors for PONV are gender (female), non-smoking patients, a history of PONV, and the use of opioids during or after surgery. The occurrence of PONV in the patients presenting a high-risk factor is $21 \%, 39 \%$ in those with two risk factors, $61 \%$ in those with three, and $79 \%$ in those with four risk factors [14]. Antiemitic drugs are recommended for high-risk patients. We therefore administered a preventive antiemitic drug, ramosetron, as all of our patients had at least three of the above-mentioned high-risk factors. Despite the use of the antiemitic drug, however, the oxycodone group showed a higher-than-expected occurrence rate of PONV (40\%) starting 4 hours after the surgery. This indicates that oxycodone was administered excessively, which signifies that the $1: 100$ ratio of fentnayl to oxycodone needs to be adjusted.

Although there was no statistically significant difference between the two groups in terms of satisfaction with the IVPCA 48 hours after the surgery (Table 4), five patients in group $\mathrm{O}$, as opposed to none in Group F, reported disappointment. These results may indicate that although the oxycodone group experienced more effective pain relief than the fentanyl group, the higher occurrence of PONV in Group O undermined the patients' satisfaction.

The present study has several limitations. First, we were not able to control the release rate, lockout interval, and volume per administration, as we used Boxter's IV-PCA in order to examine 
the total amount of analgesic consumed, which was the primary outcome of the study. As a result, the occurrence of PONV was too high, as the release rate could not be reduced even when the pain score was low enough or when the patient complained of side effects such as nausea. In the course of the study, when a patient's side effects became too severe, the IV-PCA had to be interrupted and reconnected later - once the symptoms had subsided or the patient requested it -, preventing accurate calculation of the equianalgesic dose of fentanyl and oxycodone. The second limitation was that the patients' pain thresholds were not tested before conducting the study. Finally, although the analgesic effect of opioids is related to the plasma concentration, the minimum effective concentration and minimum effective anal- gesic concentration were not measured.

In conclusion, oxycodone presented a better analgesic effect than fentanyl when used with a conversion ratio of $100: 1$, and also had economic benefits. However, it also resulted in a higher occurrence of PONV. Hence, the appropriate equianalgesic dose ratio of oxycodone to fentanyl should be determined before using it for IV-PCA.

\section{Acknowledgments}

This work was supported by the Soonchunhyang University Research Fund.

\section{References}

1. Grass JA. Patient-controlled analgesia. Anesth Analg 2005; 101(5 Suppl): S44-61.

2. Momeni M, Crucitti M, De Kock M. Patient-controlled analgesia in the management of postoperative pain. Drugs 2006; 66: 2321-37.

3. Pöyhiä R, Vainio A, Kalso E. A review of oxycodone’s clinical pharmacokinetics and pharmacodynamics. J Pain Symptom Manage 1993; 8: 63-7.

4. Pöyhiä R. Opioids in anaesthesia: a questionnaire survey in Finland. Eur J Anaesthesiol 1994; 11: 221-30.

5. Koch S, Ahlburg P, Spangsberg N, Brock B, Tonnesen E, Nikolajsen L. Oxycodone vs. fentanyl in the treatment of early post-operative pain after laparoscopic cholecystectomy: a randomised double-blind study. Acta Anaesthesiol Scand 2008; 52: 845-50.

6. Woodhouse A, Hobbes AF, Mather LE, Gibson M. A comparison of morphine, pethidine and fentanyl in the postsurgical patient-controlled analgesia environment. Pain 1996; 64: 115-21.

7. Joshi GP. Multimodal analgesia techniques and postoperative rehabilitation. Anesthesiol Clin North America 2005; 23: 185-202.

8. Ordóñez Gallego A, González Barón M, Espinosa Arranz E. Oxycodone: a pharmacological and clinical review. Clin Transl Oncol 2007; 9: 298-307.

9. Lalovic B, Kharasch E, Hoffer C, Risler L, Liu-Chen LY, Shen DD. Pharmacokinetics and pharmacodynamics of oral oxycodone in healthy human subjects: role of circulating active metabolites. Clin Pharmacol Ther 2006; 79: 461-79.

10. Kalso E. Oxycodone. J Pain Symptom Manage 2005; 29(5 Suppl): S47-56.

11. Silvasti M, Rosenberg P, Seppala T, Svartling N, Pitkanen M. Comparison of analgesic efficacy of oxycodone and morphine in postoperative intravenous patient-controlled analgesia. Acta Anaesthesiol Scand 1998; 42: 576-80.

12. Pedersen KV, Olesen AE, Drewes AM, Osther PJ. Morphine versus oxycodone analgesia after percutaneous kidney stone surgery: a randomised double blinded study. Urolithiasis 2013; 41: 423-30.

13. Pereira J, Lawlor P, Vigano A, Dorgan M, Bruera E. Equianalgesic dose ratios for opioids. a critical review and proposals for long-term dosing. J Pain Symptom Manage 2001; 22: 672-87.

14. Apfel CC, Laara E, Koivuranta M, Greim CA, Roewer N. A simplified risk score for predicting postoperative nausea and vomiting: conclusions from cross-validations between two centers. Anesthesiology 1999; 91: 693-700. 